\title{
Control de la Mosca Linterna con Manchas (Spotted Lanternfly- SLF) en Hogares de Virginia
}

\author{
Escrito por Andy Dechaine, Eric Day, y Douglas Pfeiffer, Entomology, Virginia Tech \\ Mark Sutphin y Beth Sastre, Agentes Extensionistas, Virginia Cooperative Extension
}

\begin{abstract}
Introducción: Spotted Lanternfly (SLF) se ha establecido rápidamente como una plaga agrícola, alimentándose principalmente de uvas y árboles frutales, al igual que de muchas especies de maderas duras. Como resultado, en cualquier que SLF se ha establecido, se ha convertido en una gran plaga residencial.
\end{abstract}

Daño: Cuando SLF se encuentra en una etapa inmadura, las ninfas tienden a alimentarse de nuevas y más pequeñas secciones de crecimiento de árboles y parras (Figura 1). Los árboles jóvenes con infestaciones abundantes muestran síntomas de ablandamiento, marchitez y muerte de ramas (Figura 1). Cuando se encuentra en la etapa adulta, SLF se alimentará de cualquier parte del árbol y puede causar heridas negras y llorosas a lo largo del tronco. Al alimentarse, exuda un producto de desecho llamado melaza (honeydew) (Figura 2). La melaza tiene un alto contenido de azúcar y se vuelve pegajosa a medida que recubre la superficie. Los depósitos continuos de melaza en el suelo, en la vegetación circundante y en otras superficies; como el patio, eventualmente conducirán al crecimiento del moho hollín (sooty mold) (Figura 2).

Hospederos: Se ha informado que SLF se alimenta de hasta 70 diferente especies hospederas. Éstas incluyen: arce, arce rojo, arce plateado, arce azucarero, zumaque liso, almez, falsa acacia, acacia de tres espinas, nuez negra, nogal de castilla, fresno, olmo, roble, sicómoro, magnolia meridional, sasafrás, árbol de las abejas, árbol de mimosa, árbol del cielo, morera blanca, cereza, manzana, durazno y parras. Otras plantas incluyen: madreselva de arbusto, madreselva japonesa, hiedra venenosa, hiedra inglesa, rosa ornamental, rosa multiflora, bardana, mostaza de ajo, enredadera de Virginia y lúpulo.

Control y Manejo en Hogares: Se espera que este insecto continúe expandiendose e impacte áreas residenciales. Para los propietarios de viviendas, SLF generalmente será una plaga molesta ya que se alimenta de la savia de las plantas y deposita melaza en las superficies circundantes. Es importante que los propietarios tengan la información que necesitan para ayudar a reducir el impacto y la propagación de este insecto.

Cortar y Tratar: El árbol del cielo (Ailanthus altissima) (TOH) es una especie de árbol invasiva nativa del mismo lugar que SLF y se sabe que es la especie hospedera preferida para todas las etapas de la vida. Los propietarios pueden identificar y eliminar esta especie de árbol. El árbol del cielo cerca de una casa debe cortarse y tratarse con un herbicida para evitar rebrotes. Pueden ser necesarias múltiples aplicaciones para matar completamente el árbol. Esta acción, indirectamente disminuirá la deposición de la melaza y el desarrollo del moho hollín en la casa, el patio, etc. Si no está seguro si tiene el árbol del cielo o el árbol es grande, comuníquese con un arborista o con el personal de extensión del condado para obtener asesoramiento.

Insecticida: Hay varios insecticidas disponibles para matar SLF, estos pueden ser de dos tipos. Los insecticidas de contacto: matarán a SLF cuando se rocía directamente sobre el insecto, o cuando el insecto camina sobre una superficie que contiene residuos de insecticida. Los insecticidas de contacto deben aplicarse a las superficies de los árboles donde se encuentra SLF alimentándose y caminando. Limite las aplicaciones a los árboles en donde SLF se encuentra activamente. Su reproducción es de mayo a octubre. Empiece a partir de mayo y repita según sea necesario. Imidacloprid, carbaril, pyrethrin o bifenthrin están aprovados en Virginia para el control de SLF en árboles y arbustos. Verifique la etiqueta del insecticida, SLF no necesita estar en la etiqueta, pero la planta en la que se encuentra sí. Asegúrese de usar productos etiquetados para árboles y arbustos cuando trate plantas de jardín o etiquetados para frutas o uvas cuando lo use en esas plantas.

Los insecticidas sistémicos: son absorbidos por el árbol y el insecto recibe una dosis cuando se alimenta de la savia del árbol. Estos insecticidas se aplican en la tierra alrededor de la base del árbol, en la corteza o se inyectan en el árbol cerca de la base. Se recomienda inyectar el árbol, ya que este método limitará la exposición humana al insecticida. Sin embargo, la inyección de un árbol se hace mediante un equipo especial por lo que requiere personal calificado.

Es importante tener en cuenta los insectos benéficos al decidir aplicar un insecticida. La aplicación debe hacerse después de la temporada de floración. Esto limitará el impacto negativo no deseado a los polinizadores y a otros insectos benéficos como las abejas melíferas. Póngase en contacto con el personal de extensión local o arbolistas profesionales para obtener recomendaciones. 
Control Biológico: No hay enemigos naturales efectivos en este momento.

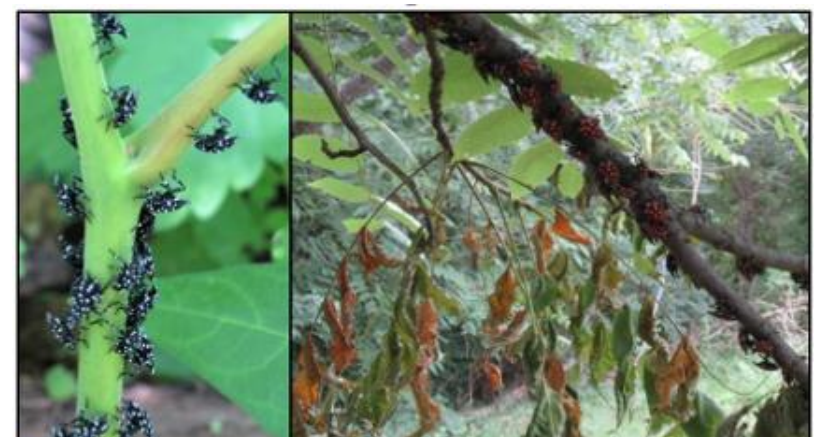

Foto 1 de la izquierda: Ninfas alimentándose de hojas tiernas del TOH. Foto de la derecha: Ninfas en el cuarto estadio alimentándose de Nogal negro (Black walnut), note las hojas marchitas; Andrew Dechaine.

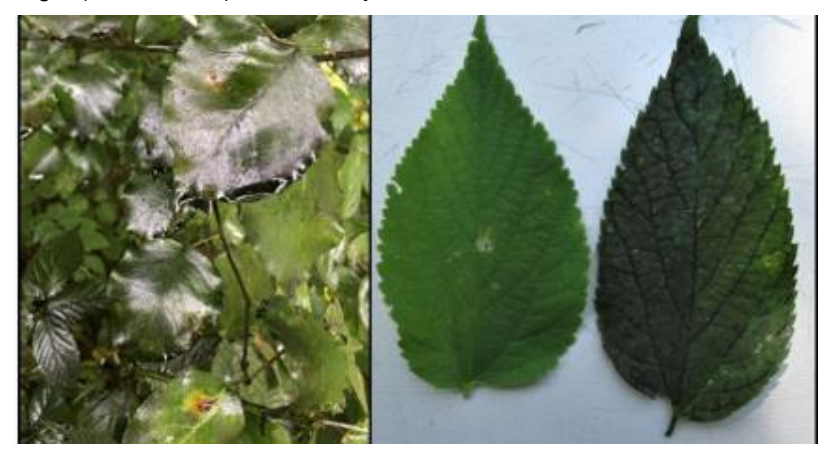

Foto 2 de la izquierda: Hojas con apariencia grasosa y brillosa resultado de la melaza; Mark Sutphin. Foto de la derecha: Hojas del Almez Americano (Hackberry) con y sin moho; Andrew Dechaine.

Dispersión lenta: Esté atento a las masas de huevos. Se cree que la forma principal en que este insecto se extenderá grandes distancias es a través del transporte humano no intencional. Como se dijo, SLF pondrá sus huevos en muchas superficies que no sean árboles. Esto podría significar leña, asadores, equipo de jardinería y escombros, muebles de jardín, botes de basura, puestos de caza y persianas, cajas, y hasta las cajas traseras de las camionetas. Si vive o visita un área infestada con SLF, asegúrese de inspeccionar su vehículo y los objetos mantenidos afuera para evitar la propagación involuntaria. Si encuentra masas de huevos, quítelos de la superficie y destrúyalos. Se puede usar una vara, una tarjeta de crédito, un destornillador, espátula o navaja de bolsillo, etc. Los huevos pueden ponerse en alcohol, desinfectante de manos, quemarse o simplemente aplastarse con el zapato.

Se pide a los propietarios a inspeccionar sus vehículos y carga durante todo el ciclo de vida de SLF antes de salir de Winchester o Frederick County.

Distribución en Virginia: SLF es una especie de insecto recién introducida en los Estados Unidos que se descubrió por primera vez en el condado de Berks, Pensilvania, en el 2014. Este insecto invasivo es originario de China y desde su introducción se ha extendido a otros 14 condados en PA y también se ha encontrado en New Jersey, Delaware y Virginia. A partir de julio del 2019, la distribución actual de SLF en Virginia está restringida a la ciudad de Winchester y al cercano condado de Frederick.

Biología e Identificación: La mosca linterna con manchas (Spotted lanternfly- SLF-) pasa por tres etapas de vida con una sola generación por año, hibernando en huevo. Las masas de huevos se depositan en el otoño y se pueden encontrar en diferentes tipos de superficies, pero generalmente en árboles con corteza lisa (Figura 3). Las masas contienen de 30-50 huevos que generalmente están cubiertos por una capa cerosa de color marrón y miden de 1-1.5 pulgadas de largo y de 0.5-0.75 pulgadas de ancho. Los huevos comienzan a eclosionar a fines de abril y continúan hasta mayo. Una vez que esto sucede, SLF pasa por cuatro etapas ninfales conocidas como estadíos. Las ninfas del primer, segundo y tercer estadio crecen progresivamente, son negras con manchas blancas en el cuerpo y las piernas (Figura 3). Las ninfas del cuarto estadio comienzan a emerger a fines de junio y pueden identificarse por su color rojo distintivo con manchas blancas y negras (Figura 3). Los adultos de SLF emergen durante julio y miden aproximadamente 1 pulgada de largo y $1 / 2$ pulgada de ancho (Figura 3 ). Los adultos comienzan a poner huevos en septiembre y sobreviven hasta noviembre, cuando las temperaturas invernales los matan.

Lycorma delicatula (Blanco) (Hemiptera: Fulgoridae).

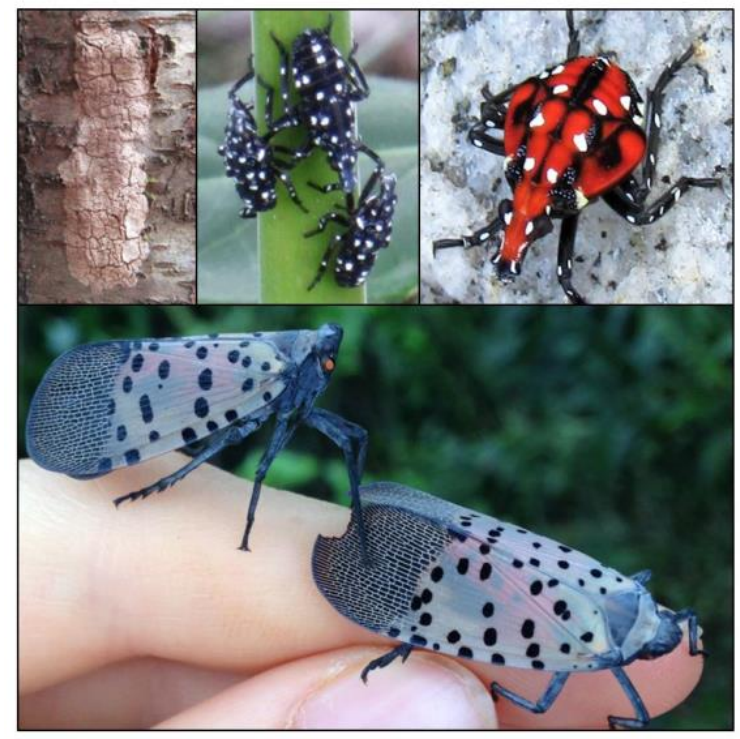

Foto 3 superior izquierda: masa de huevos. Foto de en medio: Estados ninfales del primero al tercero. Foto superior derecha: Cuarto estado ninfal. Foto inferior: Adulto de SLF; Andrew Dechaine.

\section{Información Adicional y Reporte de SLF:}

Para obtener información sobre Spotted Lanternfly, consulte: ext.vt.edu/spotted-lanternfly.

Para reportar sospechas de SLF, vaya a https://ask.extension.org/groups/1981/ask

Además, puede comunicarse con su oficina local de Virginia Cooperative Extension. 